\title{
Ultrasound-guided release of post-arthroscopy extra-articular hip adhesions in femoroacetabular impingement: a novel technique
}

\author{
Santhosh M V Reddy ${ }^{1} \cdot$ Olufemi Ayeni $^{2} \cdot$ Sri Sannihita Vatturi ${ }^{3} \cdot$ Hang Yu $^{4} \cdot$ Hema N Choudur $^{1}$
}

Received: 6 July 2020 / Revised: 20 March 2021 / Accepted: 22 March 2021 / Published online: 12 April 2021

(C) ISS 2021

\begin{abstract}
Objective To explore a novel ultrasound-guided injection technique of releasing extra-articular anterior hip adhesions in arthroscopically treated femoroacetabular impingement patients.

Materials and methods IRB-approved pilot study included 24 consecutive patients referred for ultrasound-guided injection (Jan 1, 2017-Mar 31, 2018) with anterior hip pain and/or limited hip flexion following arthroscopic treatment for femoroacetabular impingement. They underwent ultrasound-guided pressure injection, releasing post-operative extra-articular adhesions between the joint capsule and flexor tendons along the arthroscopic portal followed by steroid instillation. A visual analogue pain score of 0-10 and standard Hip Outcome Score was used to record treatment response pre-injection, at 6 weeks and at 6 months following injection. Results Nine out of 11 patients (complete data group) who answered all questionnaires showed response to injection with improvement in Hip Outcome Scores at 6 weeks and 6 months. Three out of 10 patients (incomplete data group) showed significant response to injection from clinical follow-up records. No records were available in 3 patients. In total, 12 (57.14\%) out of 21 patients showed response to injection. No post-procedure complications.

Conclusion Ultrasound-guided pressure injection is a novel, safe, and effective procedure providing pain relief and improved hip movement as evaluated in our small sample study. The limitations of this pilot project include small sample size, lack of control group, short 6-month follow-up, and single institution study. This minimally invasive technique could be a cost-effective alternative to surgical adhesiolysis and can potentially be extended for extra-articular adhesions at other peripheral joints, amenable to ultrasound.
\end{abstract}

Keywords Ultrasound-guided injection $\cdot$ Femoroacetabular impingement $\cdot$ Extra-articular hip adhesions $\cdot$ Hip arthroscopy

\section{Introduction}

Femoroacetabular impingement is the result of a size and shape mismatch between the femoral head-neck and the acetabulum. The impingement or friction between these surfaces can lead to structural damage such as labral and cartilage tears

Santhosh M V Reddy

sunnymvu99@yahoo.co.in

Olufemi Ayeni

femiayeni@gmail.com

Sri Sannihita Vatturi

sannihita.vatturi@gmail.com

Hang Yu

henry.yu@medportal.ca

Hema N Choudur

hnalinic@yahoo.com in the hip joint. This condition is treated surgically by open surgical or arthroscopic procedures with corrective osteochondroplasty and labral repair $[1,2]$.

The hip joint is enveloped by a capsule which is attached to the rim of the acetabulum and the neck of femur. Three main ligaments which strengthen the capsule-ligamentous complex

1 Department of Radiology, Hamilton General Hospital, McMaster University, 237 Barton St. East, Hamilton, Ontario L9L 2X2, Canada

2 Department of Orthopedic Surgery, McMaster University Medical Centre, 1200 Main St West, 4E15, Hamilton, Ontario L8N 3Z5, Canada

3 Department of Radiology, University of Ottawa, 75 Laurier Ave E, Ottawa, Ontario K1N 6N5, Canada

4 Department of Radiology, University of Manitoba, GA216-820 Sherbrook Street, Winnipeg, Manitoba R3T 2N2, Canada 
include iliofemoral, ischiofemoral, and pubofemoral ligaments. Standard hip arthroscopy portals include an anterolateral portal (just anterior to the tip of the greater trochanter), posterolateral portal (posterior to the tip of the greater trochanter), and an anterior portal (lateral and distal to the intersection of a line drawn transversely from the greater trochanter and a line drawn distally from the ASIS) [3]. The pathway of the anterior portal penetrates the muscle belly of the sartorius and the rectus femoris before entering the anterior capsule of the hip joint (Fig. 1). The anterior portal has been described with several variations as injury to the lateral femoral cutaneous nerve is a potential associated complication.

Post-operative pain following surgery for femoroacetabular impingement has been attributed to formation of intraarticular adhesions, extra-articular adhesions along the scope portal, repeat injury to intra-articular structures, or incomplete correction of femoroacetabular impingement bony morphology amongst other causes [4]. The intra-articular adhesions can occur between repaired portion of labrum and adjacent overlying capsule or slightly inferiorly, between femoral neck and overlying capsule. The extra-articular adhesions can occur along the arthroscopy portal tract along the anterior hip, between the external surface of joint capsule and overlying flexor tendon sheath with resultant loss of plane of separation between these structures (Figs. 2, 3, and 4).

The post-scope adhesions could result in anterior hip pain and limited range of movement [5]. While the role of and treatment for intra-articular adhesions with repeat surgery [5, 6] has been reported, there is limited information about extra- articular adhesions in literature [4]. We describe the technical details and our initial experience with a percutaneous minimally invasive ultrasound-guided pressure injection technique for treating these extra-articular adhesions. To our knowledge, this treatment option has not been explored previously in posthip arthroscopy patients and is therefore a novel technique.

\section{Materials and methods}

Institutional research ethics board approval was obtained for this prospective pilot study. Informed verbal consent was obatined from all patients.

\section{Patients}

Between Jan 1, 2017, and Mar 31, 2018, 24 patients (15 female and 9 male, ages 21 years 8 months to 51 years 1 month) with anterior hip pain and/or limited hip movement following hip arthroscopic treatment for femoroacetabular impingement were clinically evaluated and referred by a femoroacetabular impingement specialist orthopaedic surgeon to our radiology department for a preliminary hip ultrasound. The loss of range of motion in all planes more than $5^{\circ}$ compared to the contralateral side was used as a clinical test by the referring clinician in addition to lack of any other clinically explainable cause/ causes of pain or limitation of hip movements. None of the patients had post-operative MRI, prior to the ultrasound assessment. The average time interval between surgery and
Fig. 1 Sagittal MRI image with solid white arrow showing the pathway of the anterior portal of hip arthroscopy through the sartorius, rectus femoris, and then entering the anterior capsule of the hip joint. (AIIS anterior inferior iliac spine, GT greater trochanter)

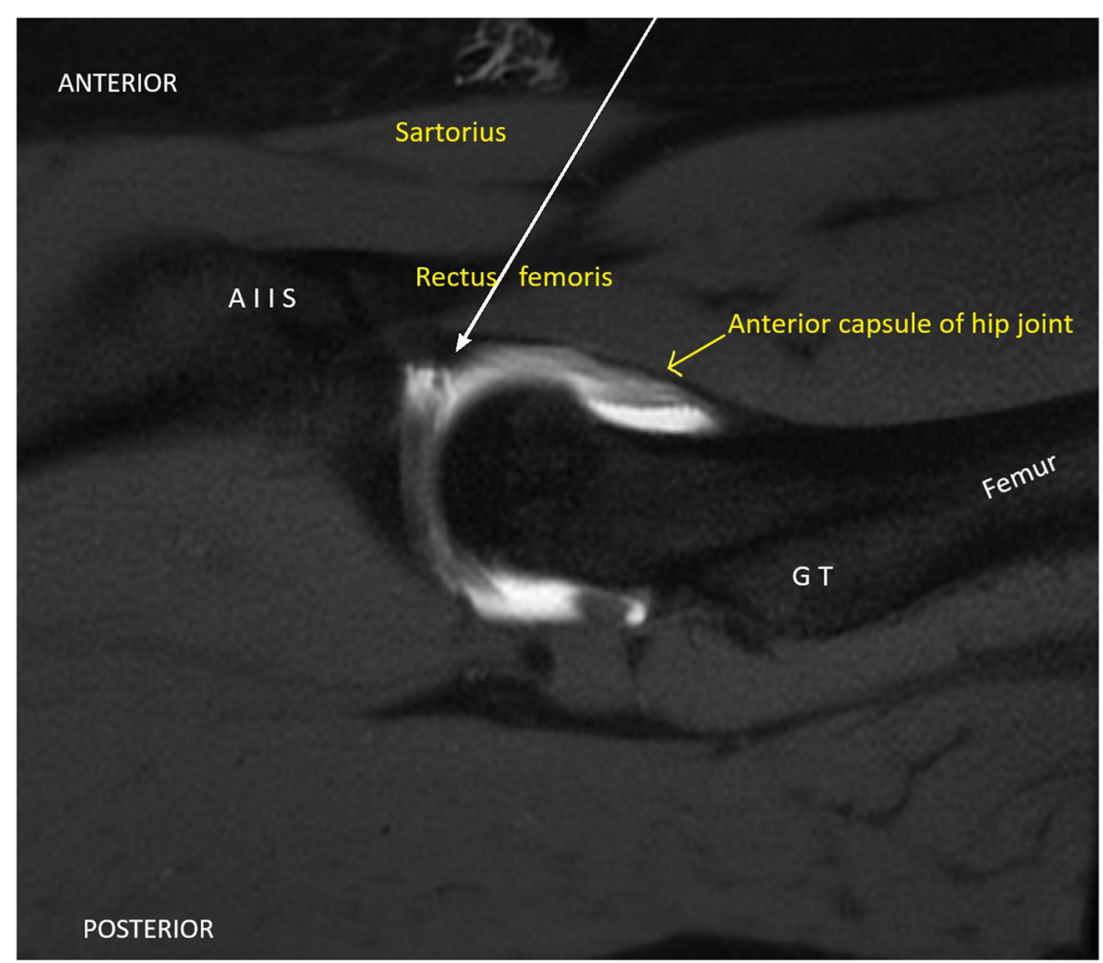


a

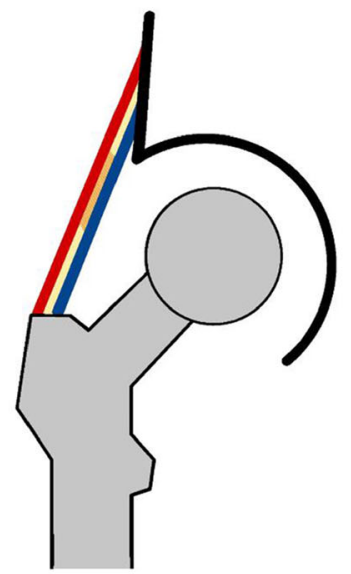

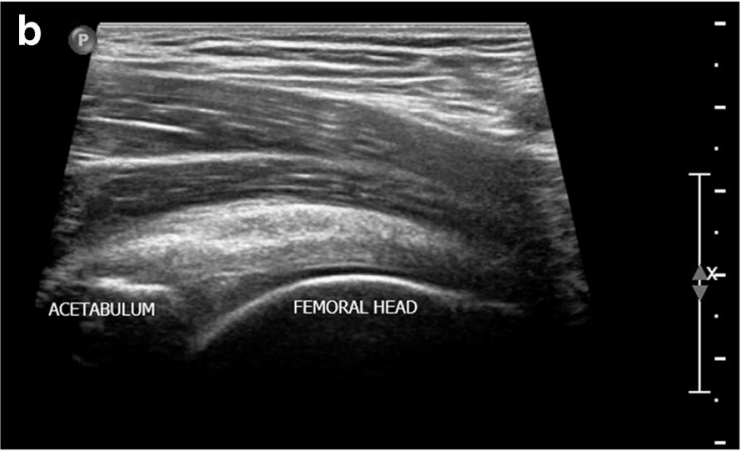

Fig. 2 Line diagram (Fig. 2a) with black=acetabulum; grey= femoral head, neck, trochanteric region; blue = joint capsule; red = flexor tendons; yellow $=$ plane between the capsule and tendons; orange $=$ zone of extraarticular adhesions along the anterior portal tract of hip arthroscopy. Long axis ultrasound images (Fig. 2b, Fig. 2c) of anterior hip joint in a 31-year-

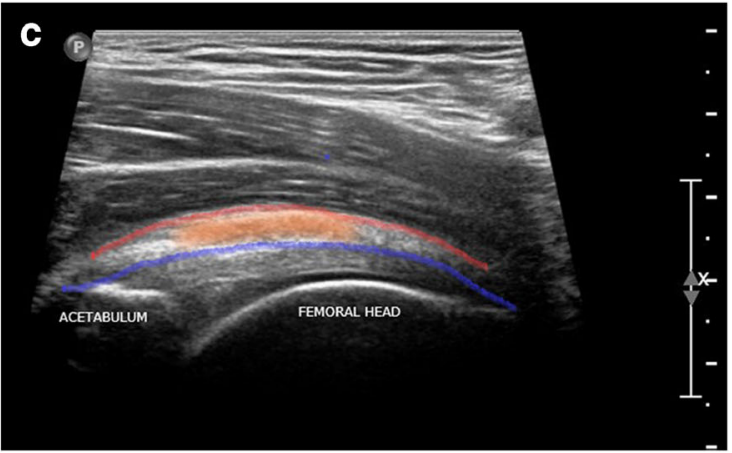

old man showing echogenic thickened extra-articular adhesions (shaded orange zone in Fig. 2a, c) between the echogenic line of hip capsule (outlined by blue line in Fig. 2a, c) and flexor tendon deeper margin (outlined by red line in Fig. 2a, c) along anterior portal tract of hip arthroscopy

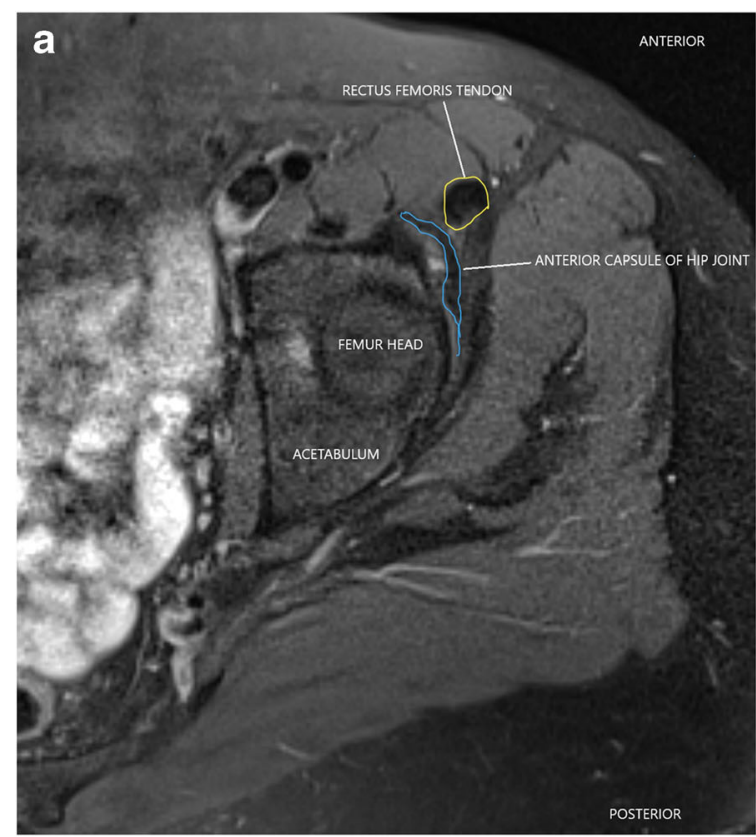

Fig. 3 Axial T2 fat saturated MRI image of hip in a normal 47-year-old patient demonstrating preserved tissue plane between the normal anterior capsule of hip joint (outlined by blue lines) and rectus femoris tendon

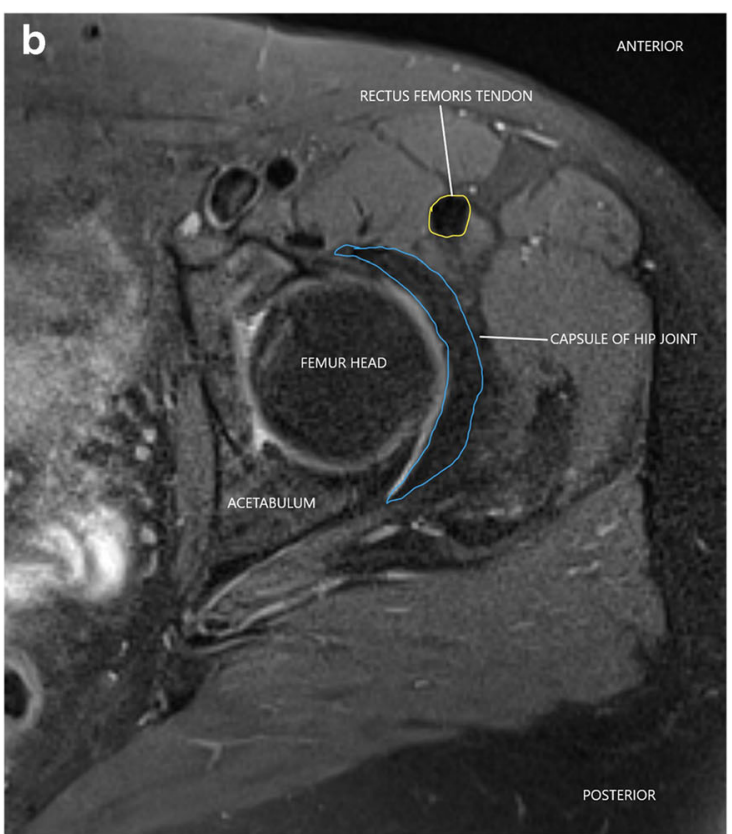

(outlined by yellow line) at level of superior aspect of hip joint (Fig. 3a) and at a slightly inferior (Fig. 3b) level 

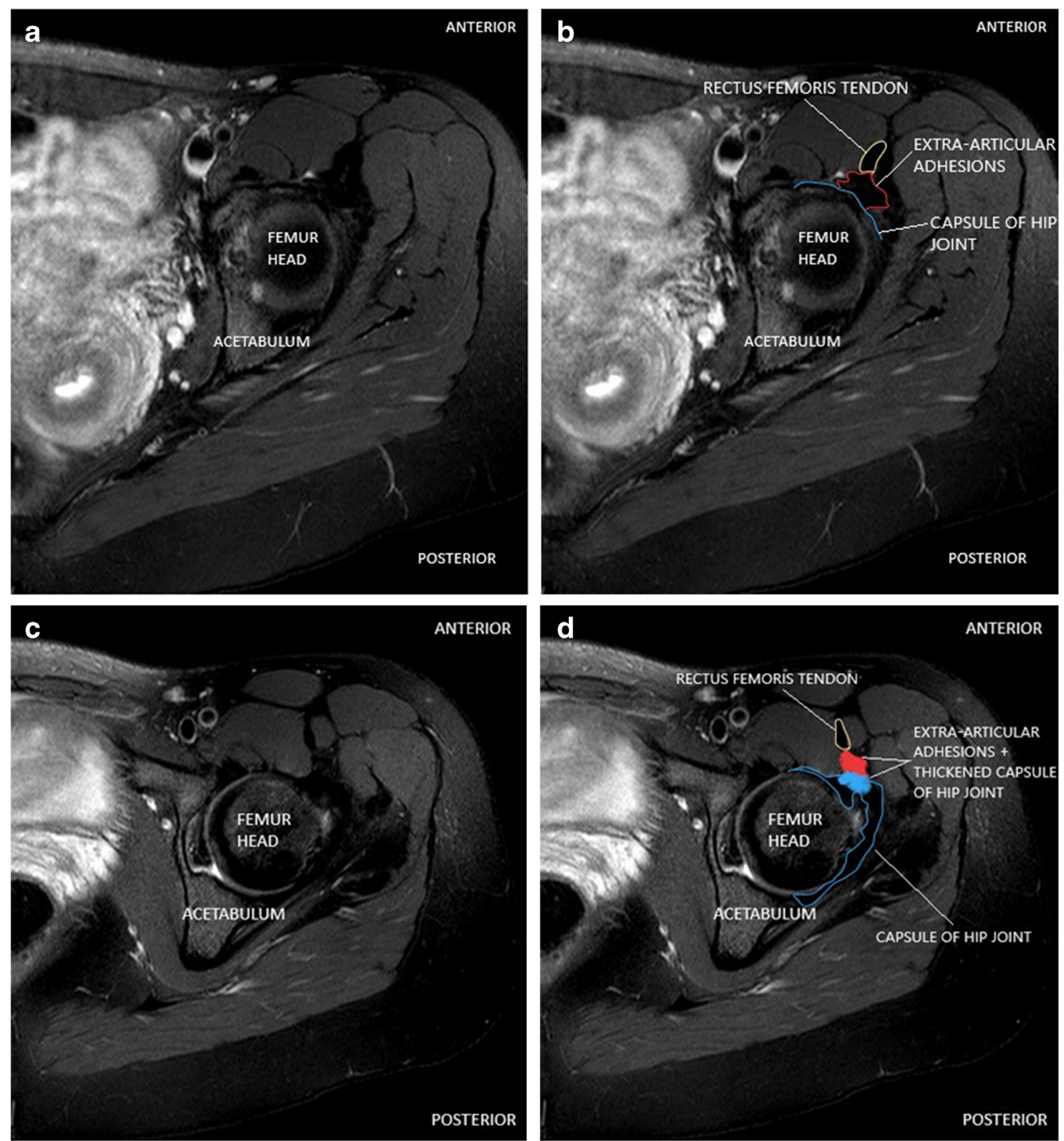

Fig. 4 Axial T2 fat saturated MRI image hip in a 27-year-old female patient demonstrating rectus femoris tendon (outlined by yellow line), capsule of hip joint (outlined by blue line), and extra-articular adhesions (outlined by red line) with loss of tissue plane (shaded blue and red areas

in Fig. 4d) between these structures along the anterior portal tract of hip arthroscopy compared to preserved tissue plane at similar levels in Fig. 3a and $b$

ultrasound intervention was 8.7 months, range 1-18 months (exception of 1 patient, 7 years post-surgery).

\section{Diagnostic ultrasound}

All the hip ultrasound examinations were performed on a Philips iU22 ultrasound machine using mainly $12-5 \mathrm{MHz}$ linear transducer and using $9 \mathrm{MHz}$ linear array in a few large patients. The diagnostic ultrasound was performed by either of the 2 musculoskeletal radiologists with 17 years and
2 years of musculoskeletal radiology experience. The arthroscopy portal was identified as extra-articular, anterior, and inferior to the repaired labrum. In our study group, ultrasound demonstrated thickened echogenic tissue between the hip capsule and iliopsoas or rectus femoris tendons with loss of separating plane between them, along the anterior arthroscopic portal tract, which was identified as extraarticular adhesions (Fig. 2). All patients described point pain and/or tenderness in this region. Comparison was made with the opposite normal side. There was inter-observer 
agreement between the two radiologists regarding the diagnostic ultrasound findings in all cases.

Consecutive patients with demonstrable extra-articular adhesions on ultrasound were then scheduled for ultrasoundguided injection.

\section{Injection technique}

All injections were performed in an outpatient radiology setting in a University-affiliated hospital by the 2 musculoskeletal radiologists. The long axis plane images on ultrasound best demonstrated the tissue planes and the adhesions along the portal tract. The injections were performed under aseptic precautions utilizing an anterolateral long axis approach with the patient in a supine position. Under direct ultrasound vision, sufficient local anaesthetic (1\% Xylocaine) agent was instilled up to the surface of the thickened echogenic tissue between the anterior hip joint capsule/flexor tendon sheath (iliopsoas or rectus femoris tendons) interface. Using a 22- or 25-gauge needle, precautions were taken to prevent penetration of the joint capsule with resultant inadvertent intra-articular injection.

After the needle tip was localised to the plane between the joint capsule and overlying flexor tendon sheath (Fig. 5), the bevel of the needle was slightly rotated to confirm that there was resistance felt to movement of the needle tip, indicative of thick adhesions. Then, about $2 \mathrm{ml}$ of local anaesthetic was injected to confirm that the injected fluid remained extraarticularly between the joint capsule and the flexor tendon sheath. Significant resistance was felt to this injection due to resistance from the extra-articular adhesions. Following injection, hydro dissection was carried out by pressure injection of $10-12 \mathrm{ml}$ of $0.5 \%$ Sensorcaine, until there was lack of resistance to the injection. A cleavage plane was seen with the injected fluid tracking along the surface of hip joint capsule separating it from the overlying thickened echogenic flexor tendon sheath (Fig. 6). One millilitre of methyl prednisolone (Depo-Medrol $40 \mathrm{mg} / \mathrm{ml}$ ) was then instilled over the surface of the hip joint, at the site of adhesiolysis.

\section{Data collection}

The pre- and post-procedure pain and range of hip movements were assessed to evaluate the effectiveness of the injection, using questionnaires. A visual analogue pain score of $0-10(0=$ no pain and $10=$ worst pain) was used to record pain improvement. The improvement of hip movements was recorded using the standard Hip Outcome Score (HOS), a functional measure with no questions related to symptoms. The Hip Outcome Score consists of two functional subscales of activities of daily living (ADL) and sports subscale with 19 and 9 questions in each subscale, respectively $[7,8]$. The data was recorded as time intervals, prior to the injection, at 6 weeks and at 6 months post-injection. The electronic follow-up records with the referring orthopaedic surgeon were reviewed in all the cases where the patients declined or could not complete the full set of questionnaires for the specified time periods. Also, any available MRI imaging of the patients post-ultrasound procedure for persistent pain were reviewed from the radiology PACS system.

\section{Results (summarized in Fig. 7 - flow chart)}

The data collection was considered complete for 11 out of 24 patients who answered all questionnaires. In these 11 patients
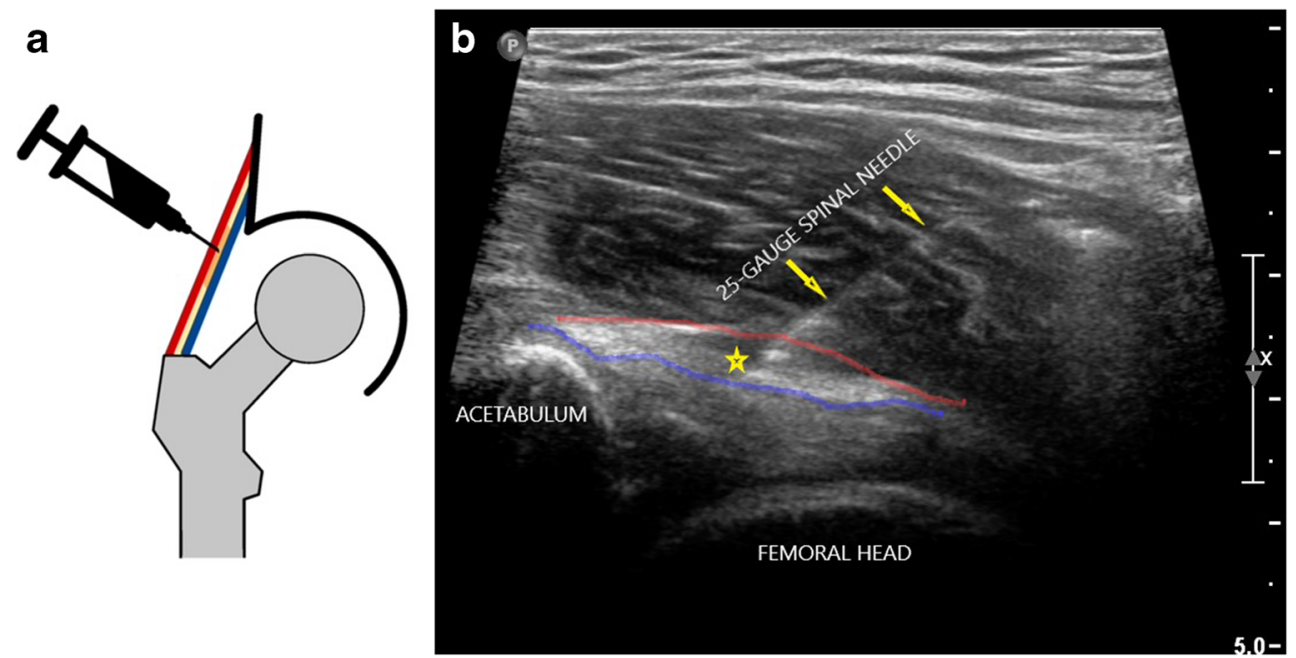

Fig. 5 Line diagram (Fig. 5a) with black=acetabulum; grey = femoral head, neck, trochanteric region; blue = joint capsule; red = flexor tendons; yellow $=$ plane between the capsule and tendons; orange $=$ zone of extraarticular adhesions along the anterior portal tract of hip arthroscopy where needle tip is positioned, and long axis ultrasound image (Fig. 5b) of anterior hip joint in a 31-year-old man showing a 25-gauge needle (yellow arrows) positioned with its tip (yellow star in Fig. 5b) in the plane between the echogenic line of hip capsule (outlined by blue line) and flexor tendon deeper margin (outlined by red line) along anterior portal tract of hip arthroscopy 
Fig. 6 Long axis ultrasound image of anterior hip injection with a 25-gauge spinal needle (yellow arrows; needle tip denoted by yellow star) in a 31 -year-old man showing release of adhesions with pressure-injected fluid (anechoic area between blue and red lines) replacing echogenic extraarticular adhesions between hip capsule (outlined by blue line) and overlying flexor tendon (outlined by red line)

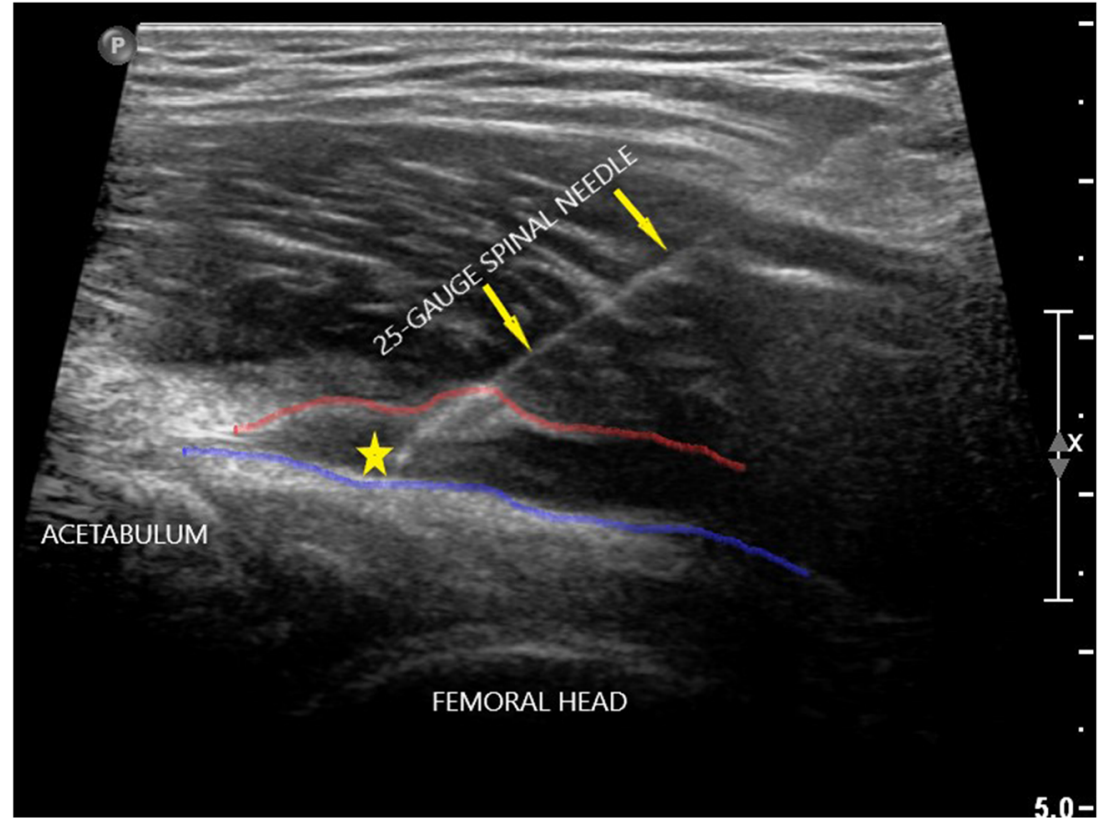

with complete data, the average improvement in pain score for all 11 patients was 2.54 at 6 weeks and 2.36 at 6 months postinjection with complete pain relief recorded in 4 patients at 6 months (summarized in Table 1 with patient demographics). The average improvement in the activities of daily living subscale of Hip Outcome Score at 6 weeks was $8.56 \%$ and at 6 months was $10.05 \%$. The average improvement in the sports subscale of Hip Outcome Score is 5.88\% at 6 weeks and $10.91 \%$ at 6 months (summarized in Table 2).

Three patients out of total 24 patients did not have any available follow-up records. The remainder 10 patients in whom electronic follow-up records were available were considered to have incomplete data set and are discussed below.

Two out of these 10 patients had significant to excellent response in symptoms documented at 6 months from the time

Table 1 Patient demographics, pain score $(0-10)$ before $=$ preinjection, at 6 weeks and 6 months from the date of injection

\begin{tabular}{llllll}
\hline & Age in years & Sex & Before & 6 weeks & 6 months \\
\hline 1 & 20 & Female & 4 & 1 & 0 \\
2 & 26 & Female & 6 & 2 & 2 \\
3 & 41 & Female & 7 & 7 & 7 \\
4 & 33 & Male & 3 & 1 & 2 \\
5 & 21 & Male & 5 & 4 & 5 \\
6 & 45 & Male & 5 & 1 & 0 \\
7 & 44 & Female & 7 & 3 & 4 \\
8 & 28 & Male & 9 & 6 & 4 \\
9 & 27 & Female & 6 & 7 & 7 \\
10 & 51 & Male & 6 & 1 & 0 \\
11 & 46 & Female & 3 & 0 & 0 \\
\hline
\end{tabular}

of injection with patients returning to all sporting activities and both were discharged from routine clinic follow-up there after. One out of these 10 patients had a partial response after 1 st injection and received a second ultrasound-guided injection 10 months from the time of the first injection. This patient had significant improvement recorded following the 2nd injection and was also discharged from routine clinic follow-up.

The remaining 7 out of 10 patients did not respond to the injection with 2 patients having transient benefit at 6 weeks only and were still symptomatic at 6 months follow-up.

In summary, 12 patients approximately $57.14 \%$ of patients ( 9 patients from the complete data set and 3 patients from the

Table 2 Hip Outcome Scores (in percentages) for ADL (ADL = activities of daily living) and sports subscale before = pre-injection, at 6 weeks and 6 months from the date of injection

\begin{tabular}{llllllll}
\hline \multicolumn{3}{c}{ ADL subscale } & & & \multicolumn{2}{l}{ Sports subscale } \\
\cline { 7 - 8 } \cline { 7 - 8 } & Before & 6 weeks & 6 months & & Before & 6 weeks & 6 months \\
\hline 1 & 73.33 & 85 & 92.64 & & 46.87 & 65.62 & 77.77 \\
2 & 77.94 & 91.17 & 95.58 & & 65.62 & 71.87 & 83.33 \\
3 & 75 & 72.72 & 72.72 & & 25 & 25 & 25 \\
4 & 67.85 & 85.71 & 82.81 & & 67.85 & 75 & 71.87 \\
5 & 96.42 & 96.42 & 89.06 & & 83.33 & 83.33 & 83.33 \\
6 & 79.68 & 92.18 & 98.43 & & 75 & 85.71 & 96.42 \\
7 & 75 & 88.33 & 84.37 & & 65.62 & 75 & 75 \\
8 & 68.75 & 89.06 & 76.56 & & 46.87 & 78.12 & 59.37 \\
9 & 70.58 & 58.82 & 55.88 & & 40.62 & 21.87 & 21.87 \\
10 & 100 & 100 & 100 & & 100 & 100 & 100 \\
11 & 82.35 & 88.23 & 100 & & 42.85 & 42.85 & 85.71 \\
\hline
\end{tabular}


Fig. 7 Flow chart outlining the results of the injection procedure and outcomes of imaging or repeat surgery in patients who did not respond to injection. $n$ number of patients. Complete data $=$ patients who completed all questionnaires. Incomplete data $=$ patients who did not complete or refused to answer questionnaires; the above information in this group was obtained from their electronic follow-up records with the referring orthopaedic surgeon and imaging results
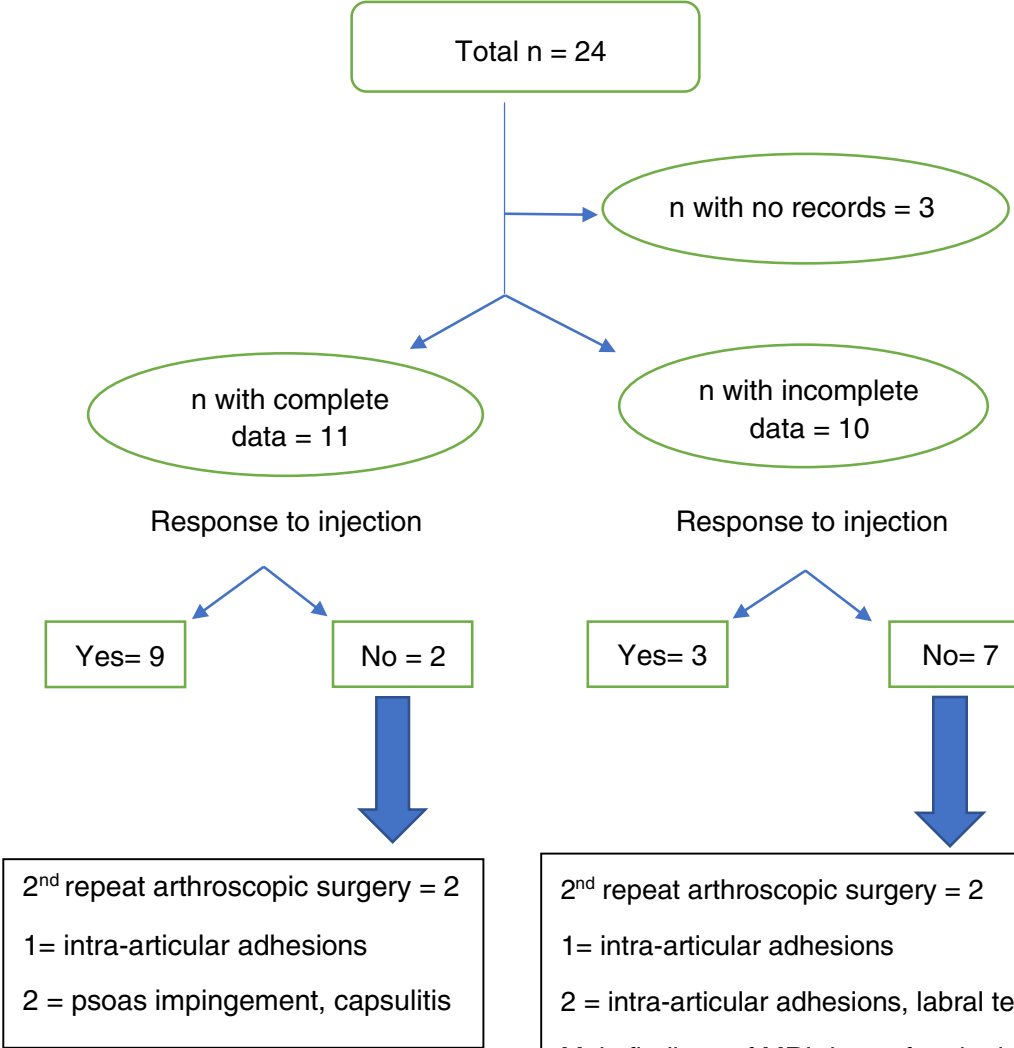

incomplete data set groups) responded to the injection while 9 patients ( 2 from the complete data set and 7 patients from the incomplete data set groups) did not benefit from the injection.

The 9 patients who had continued symptoms were investigated further with MRI or MR arthrography. Four out of these 9 patients underwent repeat arthroscopic surgery with intraoperative findings of intra-articular adhesions with labral retear in 2 patients, intra-articular adhesions in 1 patient and psoas impingement, capsulitis in the other patient. The MRI examination findings in the other 5 out of 9 patients included focal degenerative changes, greater trochanteric bursitis, fraying of labrum, and no cause was identified in 1 patient's MRI to explain the symptoms.

There were no reported complications related to the injection in our study group.

\section{Discussion}

The pathophysiology of post-hip arthroscopy adhesions has not been studied in great detail. While the topics of postsurgical intra-articular hip adhesions and extra-articular impingement have received some attention and have been described previously with relevant imaging, we found limited references for extra-articular hip adhesions in the English literature. Extra-articular adhesions cause distortion and capsular thickening, most commonly, anteriorly involving the deep aspect of psoas or rectus femoris or posterolaterally tethering the gluteus minimus or medius muscles [4]. There are no in depth or detailed imaging references regarding this topic to the best of our knowledge. In our study group, the ultrasound findings of extra-articular adhesions in one patient who did 
not respond to the injection were confirmed on a subsequent MRI following the injection and intraoperatively at the second arthroscopic surgery, confirming the presence of this entity.

The post-hip arthroscopy extra-articular adhesions result in tightening of the capsule with associated pain and resultant reduced range of movement. From the limited available previous literature, corticosteroid injections have been shown to be effective in providing significant short-term pain relief and restoration of mobility in patients with adhesive capsulitis of the glenohumeral joint, related to underlying chronic inflammation and fibrosis [9]. Corticosteroids provide symptomatic relief from reduction of pain as a result of inhibiting inflammation and prostaglandin formation. As deep fascia and tendon sheaths are both made up of fibrous connective tissue, we hypothesised that the effects would be analogous to steroid injection results in patients with adhesive capsulitis. Symptomatic hip adhesions recalcitrant to physiotherapy are traditionally treated with surgical option, usually by arthroscopic release of adhesions using a radiofrequency probe and shaver $[5,6]$. The pressure injection at the area of adhesions between the hip joint capsule and overlying flexor tendon sheath serves to release the adhesions similar to adhesiolysis at surgery.

We have explored a novel alternative ultrasound-guided intervention which can be utilised in place of the traditional surgical treatment in treating extra-articular hip adhesions. This minimally invasive percutaneous procedure is safe, technically easier, and can be performed as an outpatient procedure with a short recovery time. In this pilot study, our results using the validated assessment scoring system demonstrated the effectiveness of the procedure in improving patient's pain and function in the short-term.

The limitations to pilot study include a small sample of patients exclusively treated at one institution, lack of a control group in whom no treatment was offered, lack of a direct comparison with alternate treatment options including physiotherapy or repeat surgery, and short follow-up period of 6 months. MR and ultrasound imaging correlation of preand post-procedure extra-articular adhesions would be helpful in further understanding the role of extra-articular adhesions in symptomatic patients. In our opinion, a future research study into the role of extra-articular adhesions incorporating a more robust methodology, detailed statistical analysis, longer follow-up period, and comparison outcomes with other modalities of treatment will greatly benefit these patients, based on our experience with this pilot project using this novel approach.

\section{Conclusions}

In our pilot study, we explored the use of ultrasound-guided pressure injection followed by steroid instillation as a safe and clinically effective percutaneous, minimally invasive treatment for release of extra-articular adhesions along the anterior portal tract in arthroscopically treated patients for femoroacetabular impingement. In our opinion, this novel, minimally invasive technique can be potentially utilised to release extra-articular adhesions along scope portals at other peripheral joints amenable to ultrasound guidance.

Acknowledgements We would like to acknowledge Akhila Rachakonda for her contribution on line diagrams and figures.

\section{Declarations}

The authors declare no competing interests.

The study has IRB approval and an informed consent was obtained from all individual participants included in the study.

\section{References}

1. Leunig M, Beaule PE, Ganz R. The concept of femoroacetabular impingement: current status and future perspectives. Clin Orthop Relat Res. 2009;467(3):616-22.

2. Zhang D, Chen L, Wang G. Hip arthroscopy versus open surgical dislocation for femoroacetabular impingement: a systematic review and meta-analysis. Medicine (Baltimore). 2016;95(41):e5122.

3. Ilizaliturri VM, Valero FS, Chaidez PA, Aguilera JM. An aiming guide for anterior portal placement in hip arthroscopy. Arthroscopy. 2003;19(9):e125-e7.

4. Woodward RM, Philippon MJ. Persistent or recurrent symptoms after arthroscopic surgery for femoroacetabular impingement: a review of imaging findings. J Med Imaging Radiat Oncol. 2019;63(1): $15-24$.

5. Papavasiliou AV, Bardakos NV. Complications of arthroscopic surgery of the hip. Bone Joint Res. 2012 Jul;1(7):131-44.

6. Beck M. Groin pain after open FAI surgery: the role of intraarticular adhesions. Clin Orthop Relat Res. 2009;467:769-74.

7. Ramisetty N, Kwon Y, Mohtadi N. Patient-reported outcome measures for hip preservation surgery - a systematic review of the literature. J Hip Preserv Surg. 2015;2:15-27.

8. Martin RL, Kelly BT, Philippon MJ. Evidence of validity for the hip outcome score. Arthroscopy. 2006;22:1304-11.

9. Xiao RC, Walley KC, DeAngelis JP, Ramappa AJ. Corticosteroid injections for adhesive capsulitis: a review. Clin J Sport Med. 2017;27(3):308-20.

Publisher's note Springer Nature remains neutral with regard to jurisdictional claims in published maps and institutional affiliations. 